\title{
Emploi de la classe inversée en vue de développer la conscience phonologique et son effet sur la compréhension orale en français chez les élèves du cycle préparatoire
}

\section{Dr. Hesham Ramadan Omar}

Maître de conférences de curricula et de méthodologie du FLE, faculté de pédagogie, université d'Al Azha

\section{Ahmed Youssef El Sayed Youssef}

maître de conférences de curricula et de méthodologie du FLE, faculté de pédagogie, université Al Azharr

\section{$\underline{\text { Abstract }}$}

The present study aimed at identifying the effectiveness of using the flipped classroom strategy in developing phonological awareness and its effect on oral comprehension skills among the second year preparatory students in official language schools kalyobyia governorate. The sample of the study consisted of (60) pupils randomly selected from the preparatory second year in Ahmed Zewail official language school. They were randomly assigned into two groups: the experimental group $N=(30)$ and a control group $N=(30)$ pupils. Results of the study revealed the effectiveness of using the flipped classroom strategy in developing phonological awareness among the second year preparatory students. There was a statistically significant difference at the level of (0.01) between the mean scores of the experimental group students in the pre and posttest of the phonological awareness test and on the oral comprehension test in favor of the posttest. Also, there was a statistically significant difference at the level of (0.01) between the mean scores of the experimental group students and those of the control group students in the posttest of the phonological awareness test and on the oral comprehension test in favor of the experimental group students. Therefore, the study revealed the effectiveness of the flipped classroom strategy in developing the phonological awareness and there was a correlation relation between the phonological awareness and the oral comprehension that indicated where the phonological awareness increased, oral comprehension will be increased.

Key Words: $\quad$ Flipped Classroom-Phonological awareness-Oral comprehension Skills.

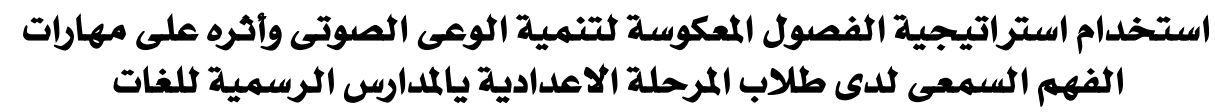

د/ هشام رمضان عمر

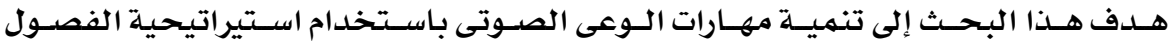

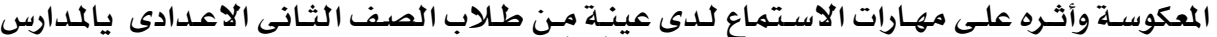

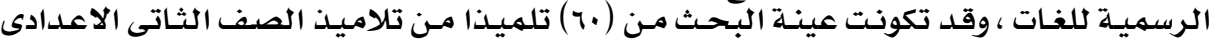

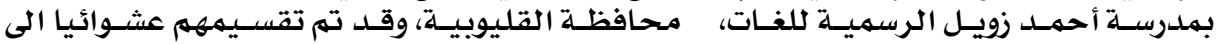

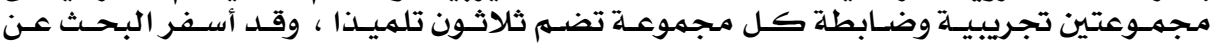

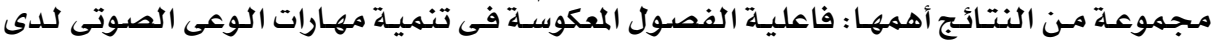




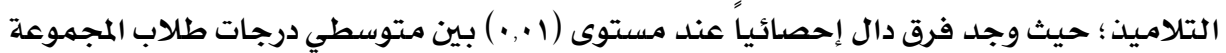

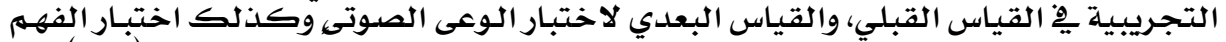

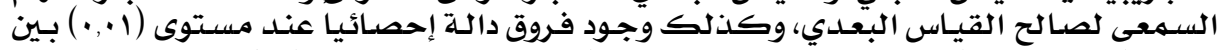

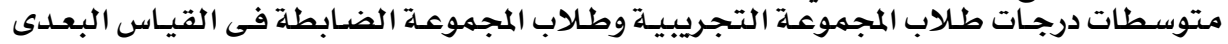

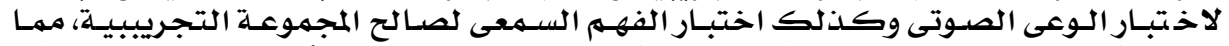

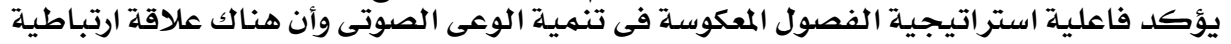

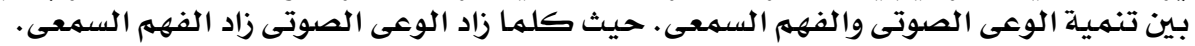
الكلمات المفتاحية: الفصول المعكوسة - الوعى الصوتى - الفهم السمعى .

\section{- Introduction:}

Les différences entre les systèmes phonologiques et phonétiques du français et de l'arabe génèrent de grandes difficultés pour les élèves arabophones en matière de compréhension et de production orales en français. Ces difficultés sont souvent plus grandes pour les élèves du cycle préparatoire car, à ce cycle, l'oral est négligé de la part des enseignants et des élèves.

La conscience phonologique peut se définir comme la capacité à manipuler et à réfléchir sur les unités phonémiques de la parole. Il s'agit de l'une des capacités métalinguistiques, la maîtrise phonologique correspond à la capacité à identifier les composants phonologiques des unités linguistiques et à les manipuler de façon délibérée (Gombert, 1990: 9)

Les études ont affirmé que les apprenants qui manifestent de bonnes compétences de conscience phonologique pourront apprendre facilement à écouter, à parler, à lire et à écrire. Voilà pourquoi les spécialistes de l'enseignement des langues étrangères sont conscients de la nécessité du développement de ces compétences chez les apprenants dès le début de leur apprentissage de la langue.

La conscience phonologique joue un rôle majeur dans le développement du langage oral. Une chose semble claire : développer la conscience phonologique améliore les compétences orales, ce qui en ferait donc plutôt un prérequis et en même temps une conséquence de cet apprentissage.

La compréhension orale est la compétence clé dans l'apprentissage d'une langue étrangère. Le développement des compétences de la compréhension orale a un effet positif sur le développement des autres compétences orales et écrites. Lors de l'écoute, l'apprenant, loin d'être passif, construit le sens d'un texte en s'appuyant sur tous les indices linguistique et non linguistique 
Comprendre n'est pas donc une simple activité de réception : la compréhension de l'oral suppose la connaissance du système phonologique, la valeur fonctionnelle et sémantique des structures linguistiques véhiculées, (Cuq \& Gruca, 2005)

Malgré l'importance de la conscience phonologique dans l'enseignement/ l'apprentissage des langues étrangères, elle n'occupe pas la place qu'elle mérite dans l'enseignement des langues étrangères en Egypte. La réalité de l'enseignement de la langue française indique que les élèves sont incapables de segmenter les mots en syllabes et en phonèmes, fusionner, soustraire, substituer les syllabes ou d'établir des liens entre la phonie et la graphie. Cette incapacité a un impact négatif sur le développement des compétences langagières surtout la compréhension orale, la compétence exige des opérations mentales plus complexes.

Tout ce qui précède nous incite à essayer de développer la conscience phonologique et à vérifier son effet sur les compétences de la compréhension orale chez les élèves du cycle préparatoire, prenant en compte de donner à chaque élève l'occasion d'apprendre d'une façon individualisée selon son rythme et sa méthode. Il est également important d'encourager le contact et l'interaction entre les élevés en utilisant des activités audio et vidéo, écoutées et regardées individuellement à la maison et pratiquées en classe dans un environnement participatif et interactif et cela peut être réalisé en classe inversée.

\section{- Problématique de la recherche :}

La problématique de cette étude réside dans la faiblesse des compétences de la conscience phonologique ce qui entrave le développement des compétences de la compréhension orale en français chez les élèves de la deuxième année préparatoire dans les écoles officielles des langues.

\section{- Le chercheur s'est assuré de ce problème à partir de:}

- son travail comme des superviseurs sur les étudiants de la section de français à la faculté de pédagogie université d'Al Azhar pendant le stage pratique. Ils ont remarqué que les élèves de la deuxième année préparatoire sont faibles en compétences de la conscience phonologique. 
- Les données des études antérieures : ces études ont montré que la plupart des élèves $d u$ cycle préparatoire sont faibles en compréhension orale

- Le résultat d'une étude pilote effectuée par le chercheur. Cette étude a indiqué que les élèves du cycle préparatoire ne maîtrisent pas la plupart des compétences de la conscience phonologique Ce qui nous a incités à utiliser la classe inversée et à vérifier son efficacité à remédier à cette faiblesse.

- De ce qui précède, on peut formuler la question principale de cette recherche:

Quelle est l'efficacité de la classe inversée sur le développement des compétences de la conscience phonologique et la compréhension orale chez les élèves de la deuxième année préparatoire dans les écoles officielles des langues?

- Cette question principale est subdivisée en questions secondaires comme suit :

- Quelles sont les compétences de la conscience phonologique nécessaires pour les élèves de la deuxième année préparatoire dans les écoles officielles des langues?

- Quelles sont les compétences de la compréhension orale nécessaires pour les élèves de la deuxième année préparatoire dans les écoles officielles des langues?

- Quel est le niveau de ces élèves dans les compétences de la conscience phonologique et celles de la compréhension orale?

- Quelle est l'efficacité de la classe inversée sur le développement de ces compétences chez les élèves de la deuxième année préparatoire dans les écoles officielles des langues?

- Est-ce qu'il existe une relation entre le développement des compétences de la conscience phonologique et les compétences de la compréhension orale chez les élèves de la première année du cycle préparatoire?

- Objectif de la recherche: Cette recherche vise à :

- Vérifier l'efficacité de la classe inversée sur le développement des compétences de la conscience phonologique et celles de la 
compréhension orale chez les élèves de la deuxième année préparatoire dans les écoles officielles des langues.

- Déterminer les compétences de la conscience phonologique et celles de la compréhension orale nécessaires aux élèves de la deuxième année préparatoire aux écoles officielles des langues.

- Développer les compétences de la conscience phonologique et celles de la compréhension orale chez les élèves de la deuxième année préparatoire aux écoles officielles des langues.

- Tester l'effet du développement des compétences de la conscience phonologique sur l'amélioration des compétences de la compréhension orale chez les élèves de la deuxième année préparatoire aux écoles officielles des langues.

- Importance de la recherche:

- Sensibiliser l'attention des responsables du système éducatif dans les écoles et les centres de formation en Egypte à l'importance de la conscience phonologique et son influence sur la performance orale chez les élèves du cycle préparatoire.

- Présenter une nouvelle stratégie pour développer les compétences de la conscience phonologique et celles de la compréhension orale en FLE.

- Aider à présenter des solutions efficaces en prenant en considération les différences individuelles des apprenants d'après la variété des activités menées en classe inversée.

- Aider les élèves à améliorer leur compétence de la compréhension orale en travaillant sur la conscience phonologique.

\section{- Limites de la recherche:}

- La recherche se limite :

- Aux élèves de la deuxième année préparatoire aux écoles officielles des langues qui étudient le français comme 2ème langue étrangère.

- Aux compétences de la conscience phonologique convenables au niveau des élèves de la deuxième année préparatoire aux écoles officielles des langues selon le CECR et la méthode Bienvenue (2).

- Aux compétences de la compréhension orale convenables au niveau des élèves de la deuxième année préparatoire. 
- Hypothèses de la recherche:

- Il existe une différence significative entre les moyennes des notes des élèves du groupe expérimental et celles du groupe témoin au post test de la conscience phonologique en faveur du groupe expérimental.

- Il existe une différence significative entre les moyennes des notes des élèves du groupe expérimental au pré-post test de la conscience phonologique en faveur du post test.

- Il existe une différence significative entre les moyennes des notes des élèves du groupe expérimental et celles du groupe témoin au post test de compréhension orale en faveur du groupe expérimental.

- Il existe une différence significative entre les moyennes des notes des élèves du groupe expérimental au pré-post test de la compréhension orale en faveur du post test.

- Il existe une relation entre le développement des compétences de la conscience phonologique et les compétences de la compréhension orale chez les élèves de la deuxième année préparatoire.

- Outils de la recherche:

- Un questionnaire adressé aux experts et aux spécialistes, en vue de déterminer les compétences de la conscience phonologique nécessaires aux élèves de la deuxième année préparatoire aux écoles officielles des langues.

- Un questionnaire adressé aux experts et aux spécialistes, en vue de déterminer les compétences de la compréhension orale nécessaires aux élèves de la deuxième année préparatoire aux écoles officielles des langues.

- Un pré - post test pour mesurer le niveau des compétences de la conscience phonologique chez ces élèves avant et après l'application du programme.

- Un pré - post test pour mesurer le niveau des compétences de la compréhension orale chez ces élèves avant et après l'application du programme.

\section{- Echantillon de la recherche:}

Dans cette recherche, l'échantillon comprend seulement des élèves de la deuxième année préparatoire aux écoles officielles des langues (école d'Ahmed Zoweil) au gouvernorat de Qalyoubiya. 
Ces élèves sont répartis en deux groupes (expérimental et témoin), chaque groupe comprend 30 élèves.

- Procédures de la recherche:

Pour répondre aux questions de cette recherche et pour vérifier ces hypothèses, le chercheur ont suivi les étapes suivantes :

- Premièrement le cadre théorique :

- La chercheuse a consulté des ouvrages qui portent sur les axes suivants :

$\checkmark$ La conscience phonologique.

$\checkmark$ La compréhension orale.

$\checkmark$ la stratégie de la classe inversée (sa définition, son importance, ses principes, ses types, les rôles de l'enseignant et ceux des apprenants).

- Deuxièmement le cadre expérimental :

- Elaborer un questionnaire portant sur les compétences de la conscience phonologique nécessaires aux élèves de la deuxième année préparatoire.

- Elaborer un questionnaire portant sur les compétences de la compréhension nécessaires aux élèves de la deuxième année préparatoire.

- Présenter les questionnaires au jury pour préciser les compétences les plus importantes à développer d'après le CERC.

- Elaborer les matériels du traitement reformulés à la lueur de la stratégie de la classe inversée (quatre unités).

- Elaborer les outils de la recherche qui comprennent : Un test de la conscience phonologique et celui de la compréhension orale.

- Soumettre ces outils de la recherche au jury et prendre en considération leurs avis et leurs modifications pour s'assurer de leur validité et pour parvenir à leur forme finale.

- Etude pilote de ce test sur un échantillon choisi, au hasard, parmi les élèves de la deuxième année préparatoire aux écoles officielles des langues.

- Calculer la validité et la fidélité du test. 
- Calculer des coefficients de facilité et de difficulté de Chaque item.

- Choisir l'échantillon de la recherche parmi les élèves de la deuxième année préparatoire aux écoles officielles des langues.et la répartition de cet échantillon en deux groupes (groupe expérimental et témoin).

- Appliquer les pré-tests sur les deux groupes de la recherche.

- Enseignement par la stratégie de la classe inversée au groupe expérimental.

- Appliquer le post test sur les deux groupes.

- Analyse statistique et interprétation des résultats des pré- post tests.

- Proposition des recommandations de cette recherche.

- Terminologies de la recherche

- La conscience phonologique :

C'est la connaissance consciente et explicite que les mots du langage sont formés d'unités plus petites, à savoir les syllabes et les phonèmes.

Elle se traduit par la capacité à percevoir et à identifier les différents composants phonologiques et à les manipuler : localiser, enlever, substituer, inverser, ajouter, combiner. (Descazaux, 2009, 1) (Desrochers, Kirby, Thompson, \& Fréchette. 2009 : 2)

\section{- La compréhension orale :}

La compréhension orale est une compétence à identifier et à comprendre ce que les autres veulent dire. Ce processus contient des éléments tels que la compréhension de l'accent et de la prononciation du locuteur, sa grammaire et son vocabulaire ainsi que la saisie de son sens (Yagang, F.1993:16).

\section{- La classe inversée}

La classe inversée est une approche pédagogique consiste à inverser et à adapter les activités d'apprentissage traditionnellement proposées aux apprenants en utilisant en alternance la formation à distance et la formation en classe pour prendre avantage des forces de chacune (Diane Leduc:2013).

\section{- Le cadre théorique}

\section{- La compréhension orale}

Écouter, c'est non seulement percevoir correctement des sons mais aussi faire un effort intellectuel pour comprendre, c'est-à-dire 
sélectionner, organiser et intégrer l'information transmise par l'émetteur. Donc l'écoute est un ensemble de comportements et d'habilités menant à la compréhension (Letarte \& Lapond, 2001: 5).

Donc l'écoute nécessite la compréhension. Sans compréhension il n'existe aucun intérêt de l'écoute

La compréhension orale est l'aptitude résultant de la mise en æuvre des processus cognitifs qui permet à l'apprenant de construire le sens d'un message qu'il écoute. L'écoute conduit, en fonction de l'objectif poursuivi, à percevoir soit de manière exhaustive tous les éléments (discrimination orale), soit de manière sélective certains de ces éléments, pour mener à une compréhension qui peut porter sur la totalité du message (totale) ou sur une partie de ce message (partielle) (Jean, 2003:50-51).

La compréhension orale est une compétence à identifier et à comprendre ce que les autres veulent dire. Ce processus contient des éléments tels que la compréhension de l'accent et de la prononciation du locuteur, sa grammaire et son vocabulaire ainsi que la saisie de son sens. (Yagang, 1993:16)

Après avoir abordé ces différents points de vue sur la définition de la compréhension orale, on peut conclure que la compréhension orale est un processus interactif ayant un aspect mental cognitif et systématique qui vise, non seulement à saisir le sens à travers des signes oraux, mais également à comprendre le fond afin de se communiquer, de critiquer, de donner des opinions, d'inférer, de créer et de déduire.

\section{- Les processus mentaux de la compréhension orale}

La compréhension orale renferme plusieurs processus mentaux à savoir :

\section{$\checkmark$ Le repérage}

Le repérage fait référence à la recherche d'information qui sont données intégralement et explicitement dans le discours oral. L'apprenant peut repérer un mot ou une phrase du discours qui correspond à l'information recherchée.

\section{$\checkmark$ La sélection}

L'information que l'apprenant recherche, se trouve dans l'ensemble d'autres données dont il n'a pas besoin. Il est convenable de sélectionner l'information qui est nécessaire à ce moment -ci. 


\section{$\checkmark$ Le regroupement}

L'information recherchée par l'interlocuteur se trouve à différents endroits dans le discours. L'apprenant doit donc repérer l'information, la sélectionner, la regrouper afin de la résumer, la comparer.

\section{$\checkmark$ L'inférence}

Peut-être l'information visée n'est pas donnée explicitement dans le discours. L'apprenant doit donc consulter ses connaissances antérieures du sujet pour découvrir l'information recherchée.

\section{$\checkmark$ L'évaluation}

L'évaluation est le processus mental qui sera utilisé par l'apprenant une fois afin de juger la pertinence de l'information écoutée, et de la comprendre profondément. Critiquer ou prendre une décision (Leduc \& Mariez 1990: 11-13).

\section{- Les étapes de la compréhension orale}

Il est favorable de segmenter la tâche de la compréhension orale en quelques étapes essentiels pour faciliter son apprentissage.

\section{$\checkmark$ La pré écoute :}

C'est l'étape de l'anticipation du contenu du discours. L'enseignant doit s'assurer que l'apprenant dispose de quelques connaissances sur le sujet à écouter. Pendant cette étape, l'enseignant vérifie le vocabulaire déjà acquis chez les apprenants et leur présente celui qu'ils ne possèdent pas et dont ils auront besoin pour comprendre.

La pré écoute est l'occasion de présenter le vocabulaire nouveau en utilisant des définitions, en donnant des traductions, en s'aidant de paraphrases, et à travers le contexte. L'enseignant peut proposer des activités concrètes comme: la manipulation des objets, l'observation des images ou de dessins, etc.

\section{$\checkmark$ L'écoute}

Dans cette étape, l'apprenant commence à aborder les informations d'une façon globale. Il tente de saisir le sens global du texte grâce aux indices qu'il a pu reconnaître pendant la première écoute. L'apprenant est appelé à comprendre la situation pour saisir le cadre dans lequel le texte se déroule: d'où provient le texte? A qui s'adresse-t-il ? Quel est son but? La deuxième écoute sert à déterminer la structure d'un message, faire une synthèse de différentes idées et en inférer d'autres, réviser un jugement, classer, etc.

\section{0}




\section{$\checkmark$ La poste écoute}

Réinvestir l'acquis dans une tâche réelle. C'est le temps d'évaluer ce que l'apprenant a compris à travers les stratégies qu'il a utilisées. Il faut pratiquer une écoute sélective conduisant à filtrer et à éliminer l'information inutile. L'apprenant doit approfondir ses connaissances en proposant une série d'activités ou de tâches réelles.

Il est utile que l'apprenant cherche des informations supplémentaires, lire des livres sur le même thème (Cornaire \& Germain, 1998 :5).

\section{- Les facteurs qui influencent la compréhension orale :}

Plusieurs variables ou facteurs peuvent effectuer la compréhension orale à savoir :

\section{$\checkmark$ Facteurs concernant les caractéristiques du locuteur.}

Les caractéristiques des interlocuteurs (les deux personnes qui échangent la parolel le discours) influencent profondément la compréhension orale: Parmi ces caractéristiques, on trouve l'habileté langagière, la connaissance de la langue, le style, les connaissances des règles socioculturelles, la personnalité, le sexe et l'âge.

\section{$\checkmark$ Facteurs concernant les caractéristiques de l'auditeur.}

- Le niveau de compétence Langagière :

En effet, il y a une corrélation étroite entre la compréhension et la compétence langagière. Les débutants distinguent mal les sons différents ou similaires, parce qu'ils ignorent les règles phonologiques. Mais à mesure que la compétence langagière s'améliore.

- La mémoire

La mémoire est un ensemble de processus biologiques et psychologiques dont la fonction générale est de coder l'information, de la stocker et de la récupérer pour pouvoir la restituer (Murieux, 1993: 128).

- Quant aux informations, Elles sont stockées sous trois formes de mémoire:

- La mémoire épisodique retient les informations sous formes de scénarios. Cette mémoire n'est jamais comble. Même un lecteur expert peut se laisser surprendre par certaines intrigues.

- La mémoire sémantique organise les informations sous une forme apparentée au dictionnaire analogique. 
- La mémoire procédurale gère essentiellement la planification. Toute ce qui concerne les actions à venir, les décisions à prendre dans une (zone tampon) sous la forme de représentation de ce qu'il faut faire" (Louise, 2005 :14).

- Le degré d'attention:

L'acquisition d'une langue étrangère est affectée par le degré d'attention. Plus un texte ou un échange est long, plus les contraintes deviennent importantes sur le plan de l'attention.

- L'affectivité:

L'affectivité est attachée aux attitudes, aux croyances, aux émotions, à la confiance en soi.

- L'âge :

Il est certain que la relation entre l'âge et la capacité d'apprendre une langue étrangère est une relation inversé.

- Les connaisseuses antérieures :

L'apprenant doit établir des liens entre ses connaissances antérieures, stockées en mémoire et les connaissances envisagées inconnues. L'apprenant doit donc savoir comment employer ces connaissances pour faciliter la compréhension et pour élargir son bagage cognitif (Cornaire, 1998: 75-89).

$\checkmark$ Facteur Propre aux caractéristiques du texte ou le message transmis:

En effet, certaines caractéristiques textuelles exercent une influence sur la compréhension orale :

- Le débit

Le débit est défini comme "la vitesse à laquelle l'orateur parle"

Lorsque le débit d'un texte augmente la compréhension diminue c'est pour cela il faut continuer à parler aux apprenants à une vitesse normale (Aouina, 2008:50).

- Les pauses :

La pause correspond à un arrêt définitif ou à une suspension de la parole pour un court laps de temps. Elle marque la fin d'un groupe rythmique. On peut dire que l'existence des pauses et hésitations facilitent le traitement du message où elles permettent à l'auditeur de bien traiter l'information déjà entendue et d'être prêt à la suivante.

\section{2}


- La longueur du texte :

La longueur du texte constitue une source de complexité plus grande que le type de texte lui-même. D'une part, Plus le message est long, plus le risque de surcharge cognitive est grand en raison de la faible capacité de la mémoire de travail. D'une autre part, Plus le niveau de l'apprenant est faible, plus vite sa mémoire de travail sera saturée, empêchant un traitement efficace du message et bloquant parfois la compréhension (Guichon, 2006:65).

- Les niveaux de la compréhension orale:

Il existe trois niveaux de compréhension orale:

\section{$\checkmark$ La compréhension globale:}

qui permet d'identifier les informations essentielles du message "de quoi parle- $t$ - on? D'où vient le message ? À qui s'adresse- $t$ - il ? Etc.

$\checkmark$ La compréhension sélective:

qui doit amener l'apprenant à chercher ou à vérifier, à la demande des informations précises permettant de faire un résumé du contenu. L'apprenant peut sélectionner/ distinguer entre les idées principales et celles secondaires.

\section{$\checkmark$ La compréhension implicite:}

qui vise à percevoir la tonalité du message, les intentions du ou des locuteurs. En bref, C'est la compréhension entre les lignes.

L'apprenant est amené à développer des savoirs et des savoir-faire correspondant à ces différents niveaux de compréhension qui exige l'acquisition de certaines stratégies cognitives et métacognitives qui concourent à faire émerger des sens (Caroline \& Jean, 2007:9)

\section{- La nature du processus de la compréhension orale :}

Selon Gremmo et Holec. (1990), Ce processus est décrit selon deux modèles différents comme suit :

$\checkmark$ Le modèle sémasiologique: (de la forme au sens) ascendant :

Le modèle séméiologique part de la forme pour trouver le sens. Il dépend du repérage du contenu.

Selon ce modèle, les processus de la compréhension orale sont:

- La discrimination auditive: l'auditeur isole la chaîne phonique du message et identifie les sons qui constituent cette chaîne.

\section{3}


- La segmentation: l'auditeur limite les mots, groupes de mots et les phrases que représentent ces mots.

- La synthèse : L'auditeur établit la signification globale du message à travers les sens des mots, groupes de mots et phrases.

Dans ce modèle, la signification du message se transmet en sens unique, du texte vers l'auditeur d'après la phase de discrimination et de segmentation, l'auditeur reconnaît des formes connus mais dans la phase de l'interprétation, l'auditeur attribue un sens à ces formes.

\section{$\checkmark$ Le Modèle onomasiologique: (du sens à la forme) descendant :}

Le modèle onomasiologique part du sens à la forme. Il part de l'expérience antérieure de l'auditeur, en d'autre terme, Il dépend des connaissances préalables liées à cette expérience comme les connaissances discursives, sociolinguistiques, linguistiques, psychologiques, référentielles et culturelles.

- D'après ce modèle, les processus de la compréhension orale sont comme suit:

- d'abord, l'auditeur établit des hypothèses sur le contenu du message en se fondant sur les connaissances dont il dispose, et sur les informations qu'il tire de ce message au fur et à mesure de son déroulement;

- parallèlement, l'auditeur établit, lors du défilement du message, des hypothèses formelles fondées sur ces connaissances des structures des signifiants de la langue dans laquelle est décodé le message

- ensuite l'auditeur procède à la vérification de ses hypothèses, cette vérification s'opère par une prise d'indices permettant de confirmer ou d'infirmer ses attentes formelles et sémantiques;

La dernière phase du processus, enfin, dépend du résultat de la vérification:

Si les hypothèses ne sont ni confirmées ni infirmées, l'auditeur suspend sa construction de signification en stockant les informations recueillies jusque-là, pour la reprendre, de manière différée, lorsque d'autre indices l'y aideront ;

Si les hypothèses sont infirmées, soit il reprend la procédure à zéro en établissant de nouvelles hypothèses, éventuellement sur la base

\section{4}


d'informations recueillies en appliquant la procédure sémasiologique. (Gremmo \& Holec, 1990 :3).

\section{$\checkmark$ Le modèle interactif:}

La compréhension orale n'est cependant pas un traitement soit descendant soit ascendant mais bien un processus d'interprétation interactif au cours duquel les auditeurs font appel à la fois à leur acquis et à leurs connaissances linguistiques pour comprendre le message (Rost, 2013).

\section{- Selon ce modèle :}

L'auditeur ne prête pas attention à tout en même temps. Il écoute de façon sélective, selon l'objectif de la tâche l'auditeur doit décider quoi écouter et par là même, quels processus activer (Rost. 2013) (Karima, 2009:276-277).

\section{- La conscience phonologique}

La conscience phonologique est la conscience de la structure segmentale de la parole qui aboutit à la conscience des phonèmes et à leur discrimination fine. Elle se traduit dans les capacités à identifier les composants phonologiques de la langue et à pratiquer des opérations sur ses composants : localiser, élever, substituer, soustraire, inverser, ajouter, combiner (Jean Jaurès. 2011 : 4) (Descazaux, 2009, 1).

La conscience phonologique est une compétence métalinguistique qui consiste à opérer des tâches délibérées sur les mots à l'oral : reconnaissances des syllabes, rimes et phonèmes ainsi que toutes les opérations sur ces unités (permuter, ôter, ajouter. .) (Briquet-Duhazé \& Rezrazi, 2014 : 121).

En tant que connaissance linguistique, jugée aussi comme compétence selon certains spécialistes de domaine de didactique des langues, cette conscience phonologique doit être fortement présente comme partie essentielle dans n'importe quel programme d'enseignement d'une langue quel que soit leur statut maternelle ou étrangère surtout dans les programmes d'enseignement de français langue étrangère due à ses spécificités de l'oral, une langue difficile à maitriser selon les non-natifs, c'est pour cela vient le rôle et l' importance de cette connaissance linguistique qui constitue la conscience phonologique chez les apprenants des langues étrangères. 
La conscience phonologique est la capacité à identifier les composants phonologiques des unités linguistiques et à les manipuler de manière délibérée (Demont \& Gombert.2007 : 51).

Il faut noter que la conscience phonographique exprime la compréhension du fonctionnement entre la langue orale et la langue écrite. Cette conscience phonographique comprend la conscience phonique guidée par des conventions de l'écrit. La conscience phonographique est la capacité à identifier les composants phonologiques de la langue et à pratiquer des opérations sur ces composants (les localiser, les enlever, les substituer, les inverser, les combiner (Dargnat, 2006 : 94).

La conscience phonographique consiste à développer la perception des unités minimales de la langue orale (les sons, les phonèmes constitués en syllabes orales) pour les transcrire en unités écrites correspondantes (les graphèmes, les syllabes écrites). On part de ce que l'apprenti connaît, l'oral, pour lui faire découvrir ce qu'il ne connaît pas, l'écrit (Feyfant \& Gaussel, 2007 :4).

La conscience phonologique est la capacité à percevoir, à découper et à manipuler les unités sonores du langage telles que la syllabe, la rime et le phonème. La prise de conscience de ces unités phonologiques, comme la syllabe et le phonème, et l'apprentissage des correspondances entre unités orthographiques et phonologiques sont essentiels à l'acquisition de la lecture et de l'écriture (Guelenne, 2013 : 6).

Il est à noter que la conscience phonologique se différencie de la phonologie au sens strict qui est l'étude des correspondances phonèmes - graphèmes. La conscience phonémique est le niveau d'abstraction de la conscience phonologique le plus élevé : c'est la capacité à analyser les phonèmes, à les manipuler, à les combiner.

\section{$\checkmark$ Importance de la conscience phonologique}

La conscience phonologique joue un rôle crucial dans l'apprentissage lenseignement des langues étrangères surtout dans la compréhension orale et écrite. Elle se traduit dans la capacité à établir des relations entre les phonèmes (constituants de l'oral) et les graphèmes (constituants de l'écrit). Le lien entre conscience phonologique et lecture s'explique par le fait que l'orthographe française est une orthographe alphabétique où les caractères " graphèmes » représentent une unité sonore " phonème ». Pour 
maîtriser le principe alphabétique, l'apprenant doit donc avoir acquis un niveau minimum de conscience phonémique.

Les concepteurs des méthodes d'enseignement ainsi que les enseignants doivent conduire les apprenants à comprendre le principe alphabétique : cela ne signifie pas qu'ils ont acquis les correspondances graphèmes/phonèmes, mais cela signifie qu'ils ont compris que l'écrit code l'oral, que des lettres ou des groupes de lettres (les graphèmes) représentent des unités distinctives de la langue orale (les phonèmes) assemblés en syllabes. "Il faut casser du sens pour le réduire en syllabe, puis casser l'unité sonore de la syllabe pour parvenir aux sons transcrits par les lettres de l'alphabet. " (Morel \& Danon Boileau, 1998 : 207).

Nous voyons donc ici l'importance portée à la conscience phonologique dans le domaine de l'apprentissage de la langue. Cette importance n'est pas anodine, si l'on retrouve autant de travaux servant à la développer dans les instructions officielles, c'est bien que celle-ci a un effet direct sur l'apprentissage des langues étrangères (Popot, 2011: $6)$.

Il est à noter que les enfants développent d'abord leur sensibilité implicite à des unités larges du langage (syllabes et rimes) avant de développer leur sensibilité explicite aux plus petites unités que sont les phonèmes. Les capacités métarimiques sont plus facilement développées par les jeunes enfants que les capacités métaphonémiques.

- Le développement des habiletés de conscience phonologique permet :

- le dépistage des apprenants qui présentent un risque de développer des problèmes d'apprentissage de la langue.

- la prévention des difficultés d'apprentissage auprès des apprenants à risque grâce à une intervention précoce

- l'amélioration des performances linguistique.

- l'amélioration de la compréhension et de l'expression orales et écrites.

- l'amélioration de l'orthographe (Stanké, 2001: 3-4) (Descazaux, 2009 : 4-5). 


\section{$\checkmark$ Les types de la conscience phonologique :}

La conscience phonologique porte sur la réflexion et la manipulation des éléments du langage oral. Ces types sont :

- Conscience syntaxique : Il s'agit de prendre conscience de la phrase comme étant constituée d'une suite organisée de mots.

- Conscience lexicale : Il s'agit ensuite de prendre conscience des mots qui composent la phrase.

- Conscience syllabique : Il s'agit de prendre conscience des syllabes qui construisent le mot. La syllabe est un groupe de phonèmes qui découpent naturellement un mot lorsqu'on le prononce. (Le phonème est la plus petite unité sonore du langage oral. C'est ce que l'on appelle couramment un son).

- La conscience métarimique : (niveau de l'attaque et de la rime)

- La syllabe peut être divisée en unités plus petites : l'attaque et la rime. L'attaque est la consonne ou le groupe de consonnes initial de la syllabe; la rime est constituée par l'ensemble des phonèmes qui suivent.

- Conscience phonémique : il s'agit de prendre conscience des phonèmes (sons) qui constituent les syllabes. Les phonèmes sont représentés visuellement par des graphèmes. Un graphème constitue la plus petite unité du langage écrit. C'est ce que l'on appelle couramment une lettre (Magnat, 2013 : 62).

$\checkmark$ Propositions didactique pour travailler sur la conscience phonologique

Pratiquement, l'enseignant doit envisager de prendre quelques mesures, on peut les résumer comme suit :

- La construction d'une planification de l'enseignement de la conscience phonologique

Cette planification doit prendre en considération les critères suivants:

Des critères relatifs à la nature des unités linguistiques (les syllabes, les rimes, les attaques, les phonèmes) et à leur position : syllabe en position initiale, interne, finale, phonèmes en attaque, en rime, en position interne. 
Pratiquement, c'est préférable de suivre les démarches suivantes :

Il faut d'abord commencer un travail sur la SYLLABE. Puis il conviendra de travailler sur LES RIMES pour aller progressivement via l'opposition ATTAQUE/RIME vers le PHONEME, très difficile avant l'apprentissage de la lecture, en particulier les consonnes. Commencer d'abord par les voyelles puis aborder les consonnes qui " chantent » longtemps (/f/, / $/ /, / r /, / s /$

De même il est plus facile de travailler sur la position initiale, puis finale et enfin intermédiaire.

- Des critères relatifs à la nature des opérations intellectuelles mobilisées en compréhension et en production. Il faut prendre soin d'alterner des tâches de :

- Comparaison d'éléments phonologiques

- Catégorisation : découvrir une règle de tri, appliquer cette règle

- Transformation d'éléments phonologiques : dénombrer, modifier dans la prosodie (accentuer une syllabe, la prolonger) ex : segmenter, fusionner, enlever, ajouter, substituer, inverser

- Une organisation en collectif

En classe, l'enseignant pourrait animer les séances portant sur la conscience phonologique en groupe-classe, aussi chaque apprenant intervient individuellement, ce qui permet de déceler les difficultés, les besoins de chacun et d'envisager des ateliers de soutien ou de renforcement lors de l'aide personnalisée. Durant ce moment de travail en groupe collectif, la communication entre les apprenants et la participation de tous est facilité pour :

- reformuler une consigne

- justifier le choix d'un apprenant

- corriger

- améliorer l'analyse d'un mot en fonction de la consigne

- la formulation des actions à mener pour réussir (le métalangage)

○ Des critères relatifs à l'utilisation des matériaux connus de tous

Il est important que les supports soient familiers pour les apprenants : privilégier le travail sur les prénoms de la classe, puis sur le nom des

\section{9}


objets de leur imagier de référence. Les cartes de cet imagier appartiennent à des catégories très connues des apprenants.

- Des critères relatifs au scénario identique pour toutes les séquences: Chaque séquence s'organise autour de trois types de tâches:

\section{a) la tâche principale}

Elle permet de rappeler ce qui a été appris lors des séances précédentes, de présenter clairement l'objectif, de donner la consigne, de traiter des exemples, de permettre à chaque enfant d'agir.

\section{b) la tâche de transposition}

Elle s'appuie sur la même compétence cognitive mais en apportant quelques variables didactiques pour réinvestir la compétence travaillée en l'adaptant à une autre situation.

c) Une tâche de transfert : mise en place de petits exercices ayant pour but d'automatiser les compétences nouvellement acquises (Descazaux, 2009 : 6-8)

\section{$\checkmark$ La compréhension orale et la conscience phonologique}

La compréhension du message auquel un individu est confronté passe par un processus complexe. L'individu traite l'information dans sa mémoire de travail en faisant appel à des représentations abstraites stockées en mémoire à long terme (Magnat. 2013 : 73).

Il est à noter que pour maitriser la compréhension du langage orale, on doit acquérir les composantes de la langue orale (les sons, les syllabes, les rythmes, les groupes syllabiques et les groupes rythmiques.....etc.). D'une part et avoir des connaissances sur les sujets de la compréhension d'une autre part. La majorité des apprenants ne comprennent pas que l'enseignant souhaite qu'ils analysent les composantes orales de la langue. Pour eux, l'objet et l'activité principale est d'accéder au sens des messages oraux (Mohammad, 2015 :27).

Evidemment, il y a des interactions entre les informations phonologiques fournies à l'oral et les connaissances sémantiques disponibles dans la mémoire. Éliminer de la liste les mots qui ne sont pas cohérents par rapport au contexte (Treiman, Clifton, Meyer, \& Wurm, 2003).

\section{0}


Il y a donc interactivité entre les informations sonores et les informations contextuelles. La rapidité du décodage dépend de l'expérience de l'apprenant. Il est possible d'identifier des mots dont les premiers phonèmes ne sont pas entendus. En effet, même lorsque certains phonèmes sont supprimés dans le flot parlé, l'auditeur est capable de restituer les phonèmes manquants. Il s'agit de la restauration phonémique (Gaonac'h \& Passerault, 2006 : 338).

Il s'ensuit que plus la conscience phonologique s'améliore plus la compréhension orale n'augmente. L'identification des phonèmes joue un rôle dans le processus de compréhension orale.

\section{- La classe inversée}

La classe inversée, en somme, consiste à inverser l'enseignement traditionnel des devoirs à la maison et des leçons en salle de classe, en présentiel. Et de mettre donc les leçons à la maison sous forme de vidéos oralisées, de capsules et les devoirs en classe, sous forme d'activités en groupe (Berberian, 2016 :18).

En théorie, ce modèle de classe inversée permet de tirer profit du présentiel et de la formation à distance et de se débarrasser des inconvénients.

La classe inversée est une approche pédagogique consiste à inverser et à adapter les activités d'apprentissage traditionnellement proposées aux apprenants en utilisant en alternance la formation à distance et la formation en classe pour prendre avantage des forces de chacune (Diane Leduc:2013).

La classe inversée inverse le schéma de l'enseignement traditionnel. L'apprenant peut poser des questions à l'enseignant pendant la réalisation des devoirs (activités en présentiel) et prend en charge son apprentissage de la théorie. Le cours devient une phase d'autoformation où l'apprenant prend un rôle actif puisqu'il décide luimême dans quel contexte il va apprendre, à quel moment, en prenant des notes ou non, en faisant des pauses dans la vidéo ou non. Il va ensuite mettre la théorie en pratique avec l'enseignant qui aura ici un rôle de médiateur et qui va pouvoir répondre aux questions des apprenants dans un but précis.

La classe inversée se définit comme une approche pédagogique dans laquelle une première exposition à la matière s'effectue de manière

\section{1}


autonome, dans une phase préalable à une phase présentielle animée par un enseignant. La partie préparatoire autonome peut s'effectuer avec différents types de ressources (livres et autres documents, sites Web, vidéos, logiciels...) et de tâches à réaliser (faire une recherche, répondre à un quizz...) (Lecoq, Lebrun, \& Kerpelt, 2016:5).

\section{$\checkmark$ Avantages de la classe inversée}

L'emploi de la classe inversée favorise le processus d'enseignement I apprentissage. On peut compter ses avantages suivants :

- Un moyen d'amplifier les interactions et les contacts personnalisés entre les élèves et l'enseignant.

- Un environnement dans lequel les acteurs changent de rôle : les apprenants prennent la responsabilité de leurs propres apprentissages sous la guidance de l'enseignant qui est devenu un accompagnateur attentif.

- Un mélange fertile de la transmission directe (j'enseigne) avec une approche constructiviste ou encore socioconstructiviste de l'apprentissage (c'est aux apprenants qu'il revient d'apprendre).

- Une classe dans laquelle les apprenants qui sont absents ne sont pas laissés "en arrière".

- Une classe où les contenus à apprendre sont accessibles tout le temps pour les révisions, les examens, la remédiation.

- Un lieu où les apprenants peuvent recevoir un accompagnement personnalisé.

- La possibilité de varier les ressources, de les personnaliser, de les combiner, de les contraster en vue d'amorcer et d'enrichir l'apprentissage en présentiel (Lecoq, Lebrun, \& Kerpelt, 2016:11).

\section{$\checkmark$ Les outils de TICE et la classe inversée}

Les TICE englobent tous les dispositifs et les outils numériques permettant la création ou la distribution de contenus de cours. Les TICE offrent de nombreuses possibilités d'enrichir un cours, de favoriser les travaux en groupe, d'individualiser la formation, de donner accès à des documents authentiques, ou encore d'échanger avec des pairs partout dans le monde entier. (Berberian, 2016 :19-20). Pour qu'une classe inversée soit efficace, l'enseignant doit se concentrer sur

\section{2}


les outils de TIC les plus faciles à utiliser. Ces outils technologiques adoptés en classe inversée, dont la vidéo, nous ont apporté beaucoup d'avantages comme :

- La relecture pour tous les points incompris, en s'arrêtant, en reprenant, en commençant aux moments désirés et utiles ;

- Une mise à jour si un cours a été manqué en salle de classe ;

- Un ajustement aux besoins d'apprentissage spécifiques et réels de chacun ainsi qu'aux différentes manières d'apprendre ;

- Une préparation à toute heure aux examens ;

- Un engagement plus important sur la voie de l'autonomie;

- Une croissance de confiance en soi plus grande par rapport au sujet d'un cours (Marchal, 2015 : 28).

\section{$\checkmark$ Rôles de l'enseignant et ceux de l'apprenant en classe inversée}

Il va sans dire que les rôles de l'enseignant et ceux de l'apprenant sont différenciés de la classe ordinaire. La classe inversée, ce n'est pas seulement "le cours en vidéo avant la séance et des exercices et applications pendant la séance ». C'est aussi un bouleversement dans les rapports aux savoirs et aux rôles tenus par les apprenants et les enseignants.

\section{- Rôles de l'enseignant}

L'enseignant prépare les activités, les débats et les quiz que les élèves fassent preuve de leurs connaissances apprises préalablement à la maison. Il est le responsable de contrôler le déroulement du cours et se montrer toujours disponible pour dissiper les doutes des élèves, clarifier les concepts et fournir les explications nécessaires. La classe inversée implique, par conséquent, de la part des enseignants, un travail de programmation, de scénarisation et d'explicitation assez précis (Becchetti-Bizot 2017 : 27).

Avec ce rôle d'accompagnement pédagogique, l'enseignant doit apporter aussi les outils nécessaires pour la réalisation de ces activités et s'assurer que tous les points de la leçon ont été traités avant de continuer. "Dans la classe inversée, l'enseignant n'est plus un expert mais, un facilitateur d'apprentissage (Lebrun/Lecoq 2015 : 74)

En classe inversée, l'enseignant est considéré comme médiateur pendant les phases d'activité. Il adopte une démarche didactique permettant à l'apprenant de devenir autonome dans la langue cible. Il doit pouvoir mettre l'apprenant en situation de communication réelle le plus souvent possible et le dispositif de classe inversée permet cette application (Marchal, 2015 : 24).

\section{3}




\section{- En bref les rôles de l'enseignant en classe inversée sont :}

- Organiser le travail à distance ;

- Choisir, construire et préparer les ressources ;

- Utiliser des ressources libres;

- Créer des ressources convenables à ses apprenants

- Organiser ses ressources : les outils du Web 2.0 ;

- Partager ses productions sous licence Creative Commons ;

- Organiser le travail à distance en groupe ;

- Animer le présentiel. (Lecoq, Lebrun, \& Kerpelt, 2016:22-31).

\section{$\checkmark$ Rôles des apprenants en classe inversée}

L'apprenant est le responsable de son apprentissage et il doit préparer chez lui le matériel envoyé par l'enseignant concernant le contenu du cours du lendemain. Ensuite, s'il y a quelques concepts à propos de l'explication qu'il n'a pas compris, il peut poser ses doutes à l'enseignant dans la classe; et en plus, aider d'autres camarades avec ce qu'il connaît déjà. L'apprenant se montre plus motivé et disposé à apprendre puisqu'il fait le travail de haut niveau cognitif en classe en travaillant de façon collaborative avec ses camarades et à l'aide de l'enseignant; et pour le travail le plus facile (regarder des vidéos, etc.), il le fait à la maison à son rythme (González Ramos, 2016 :12).

- On peut récapituler ces rôles suivants :

- Les apprenants regardent et analysent une vidéo ou un texte pour en dégager, individuellement ou en groupe, quelques arguments en faveur ou en défaveur des propos émis.

- les apprenants sont amenés à trouver, individuellement ou en groupe, des illustrations, des exemples, des applications à présenter à leurs pairs de courts textes ou des vidéos énoncent des avis contraires sur une thématique ou sur une problématique.

- Les apprenants construisent un débat sur ces controverses des rôles sont assignés aux apprenants ou à des groupes afin qu'ils proposent en classe une synthèse, des avis favorables ou défavorables, des exemples.

- Les apprenants proposent leurs solutions aux problèmes énoncés dans la vidéo ou dans le texte

\section{4}


- Les apprenants répondent à de courts questionnaires en ligne ou écrivent sur un forum, de manière à pouvoir observer ce qui est compris et ce qui ne l'est pas (González Ramos, 2016 : 20).

\section{$\checkmark$ Comment appliquer la classe inversée}

L'enseignant réfléchit aux contenus qu'il désire enseigner avec cette méthode. S'il n'opte pas pour des vidéos disponibles, il aura à préparer des capsules à partir des moyens technologiques à sa disposition : caméra numérique, logiciels de capture d'écran, tablette tactile, etc. Ensuite, il devra prévoir les activités obligatoires en classe, mais également des activités avancées pour les apprenants plus rapides (Dufour, 2014 : 9).

\section{- Avant le cours}

L'enseignant s'assure que les apprenants comprennent bien l'approche pédagogique du cours pour en assurer le succès, car cela exige une grande implication de leur part. Certains enseignants suggèrent d'y aller graduellement en proposant d'abord des lectures obligatoires qui seront suivies d'une activité en classe.

Ensuite, les contenus vus à la maison seront de plus en plus complexes, accompagnés de vidéos. Il faut que l'apprentissage en dehors de la classe soit actif, en exigeant quelquest âches complémentaires. Cela peut prendre plusieurs formes : un résumé des contenus, des questions de compréhension, une réflexion critique par rapport aux contenus, etc. L'objectif est de s'assurer que l'apprenant a pris connaissance de la matière.

\section{- Pendant le cours}

Pendant le temps de classe, l'apprenant est appelé à être actif dans ses apprentissages, en favorisant la réalisation d'activités, d'exercices ou d'expériences en laboratoire pour réinvestir le contenu vu dans les vidéos. À ce moment, l'enseignant joue un rôle de guide et de tuteur. Et enfin les évaluations sont planifiées et sans surprise (Roy, 2014 :2)

\section{$\checkmark$ Quatre dispositifs d'évaluation pour la classe inversée}

Evaluer les acquis des apprenants en classe inversée reflète les nouveautés dans le domaine de l'évaluation des langues étrangères. L'évaluation selon cette stratégie doit être variée, on ne se contente pas un seul type d'évaluation mais on propose plusieurs types d'évaluation innovants.

\section{5}




\section{- L'évaluation autogérée :}

Ce type d'évaluation propose de donner une large autonomie à l'apprenant dans le choix des questions comme dans le choix du moment de l'évaluation. Parfois, l'évaluation autogérée va jusqu'à confier aux apprenants la rédaction des questions d'examen, en tout cas elle présente une démarche formative d'évaluation.

\section{- L'évaluation par les pairs :}

Elle consiste à engager l'apprenant dans le processus de l'évaluation en le mettant en position d'évaluer le travail d'un autre apprenant selon une grille de critères. Il a été démontré que le caractère formateur de l'évaluation par les pairs réside dans l'exercice de production d'un feed-back plus que dans la réception du feed-back d'autrui.

- Le portfolio évaluatif :

Le portfolio est une collection ciblée de travaux qui montre les efforts individuels et les progrès de l'apprenant dans un ou plusieurs domaines. Cet outil d'évaluation s'inscrit dans la perspective d'un apprentissage à la fois réflexif (par le développement d'une forme d'autoévaluation) et expérientiel (par le recueil et la valorisation de ses expériences d'apprentissage). Il vise avant tout l'autonomie et la responsabilisation de l'apprenant dans son propre processus d'apprentissage.

\section{- L'évaluation en situation authentique :}

Les classes inversées se prêtent bien à des modalités d'évaluation originales. (Lecoq, Lebrun, \& Kerpelt, 2016:5). La stratégie des classes inversées interroge fortement les processus d'évaluation " ordinaire » basés sur la remémoration des connaissances et leurs applications dans un champ scolaire bien délimité. Par contre, l'évaluation d'après la class inversée permet d'aborder les évolutions attendues à propos des tâches des apprenants et les besoins en termes d'instruments d'évaluation pour les enseignants (Dumont \& Berthiaume, 2016 : 28). (Bouchlion, 2014)Top of FormBottom of Form

\section{$\checkmark$ Cinq raisons pour pratiquer la classe inversée en classe}

- Offre la possibilité aux apprenants d'écouter et de réécouter le contenu théorique s'ils en ont besoin.

\section{6}


- Favorise une responsabilisation des apprenants en les impliquant davantage dans leur apprentissage.

- Laisse plus de temps au suivi personnalisé à partir d'échanges avec l'enseignant.

- Permet davantage d'interactions entre les apprenants.

- Invite à une meilleure utilisation du temps en présence avec les apprenants (Roy, $2014: 5$ )

\section{- Le cadre expérimental}

- Premièrement : Les outils de la recherche.

- Le chercheur a élaboré quatre outils comme suit :

- Une grille des compétences de la conscience phonologique.

\section{But de la grille:}

Cette grille vise à déterminer les compétences de la conscience phonologique nécessaires aux élèves de la deuxième année préparatoire en français deuxième langue étrangère dans les écoles officielles des langues.

\section{$\checkmark$ Les sources de la grille:}

Pour élaborer la grille de compétences requises pour le public cible, le chercheur a consulté un grand nombre d'études, de travaux théoriques, de manuels d'enseignement / apprentissage du français langue étrangère. Nous citons à titre d'exemple;

- CECR (2001). Cadre européen commun de référence pour les langues. Conseil de l'Europe /Les Éditions Didier, paris.

- Compétences fondamentales pour les langues étrangères; standards nationaux de formation. (2011). Conférence suisse des directeurs cantonaux de l'instruction publique.

- Netten, J \& Germain, C. (2001). La précision et l'aisance en langue seconde ou étrangère. Synergie Corée. $N^{o} 1$. Revue de didactologie des langues étrangères.

- Niveaux de compétence linguistique canadiens. Français langue seconde pour adultes. (2012). Centre des niveaux de compétence linguistique canadiens. Ottawa (Ontario) Canada.

\section{7}


- Guelenne Ch., (2013) : La Conscience Phonologique : Dossier d'Activités, Les Planabums, Retz.

- Paquin Stéphanie, M.O.A.: Qu'est-ce que la conscience phonologique?,http://www3.csmb.qc.ca/ecoles/portals/14/Consc ience\%20phono_SP.pdf(15/1/2016).

- Swita, Anne-Marie \& autres (2001) : Exercices de conscience phonologique et d'activités métalinguistiques JUIN.

\section{$\checkmark$ La description:}

Nous avons abouti à une grille de compétences, sous sa forme initiale, elle se compose de deux parties:

La première partie contient le titre de la recherche, les objectifs et les consignes de la grille. La deuxième partie contient (16) items, répartis en quatre axes (syllabe, rime, phonème, phonie-graphie) représentant les compétences de la conscience phonologique nécessaires aux élèves de la deuxième année préparatoire en français deuxième langue étrangère dans les écoles officielles des langues.

Ce tableau montre le niveau de la conscience phonologique et le nombre d'items pour chaque niveau (axe)

\begin{tabular}{|c|c|}
\hline Niveaux ou axes & nombre d'items \\
\hline Syllabes & 6 \\
\hline rimes & 2 \\
\hline phonèmes & 6 \\
\hline Phonie- graphie & 2 \\
\hline
\end{tabular}

Le chercheur a formulé les items de cette grille en termes de comportements spécifiés, précis et mesurables, décrivant ce que les élèves doivent maîtriser. Devant chaque compétence se trouve un rubrique pour le degré de nécessité (très nécessaire, nécessaire, peu nécessaire, pas nécessaire).

Après avoir élaboré la grille des compétences de la conscience phonologique, nous l'avons soumis, sous sa forme initiale, aux membres du jury afin qu'ils :

- Vérifient la justesse de la formulation des compétences de la conscience phonologique, et de les modifier si nécessaire.

- Suppriment les compétences non nécessaires.

- Rajoutent les compétences non inclus.

\section{8}


- Vérifient que les compétences sont du niveau des élèves de la deuxième année préparatoire qui étudient le français deuxième langue étrangère dans les écoles officielles des langues.

Les membres du jury ont apprécié la grille des compétences de la conscience phonologique à l'exception de quelques remarques concernant la formulation. Ils ont supprimé les items numéro 6-12-1421. Ces items ont été supprimés parce qu'ils sont incompatibles avec le niveau de l'échantillon.

\section{$\checkmark$ Le calcul des pourcentages des compétences:}

Les compétences les plus importantes à développer chez les élèves d'après le jury sont dix-neuf compétences. Ce sont les compétences qui sont plus de $75 \%$.

Prenant en considération ces remarques, et faisant ces modifications, la grille a pris sa forme finale qui contient les compétences suivantes:

\section{- Premier axe : Syllabes}

- Segmenter des mots en syllabes

- Compter les syllabes d'un mot

- Repérer la syllabe initiale-finale d'un mot

- substituer des syllabes pour construire des mots

- soustraire des syllabes pour construire des mots

- additionner des syllabes pour construire des mots

\section{- Deuxième axe : Rimes}

- Repérer des rimes

- Produire des rimes

- Troisième axe : Phonèmes

- Segmenter les mots en phonèmes

- Compter les phonèmes d'un mot

- Repérer le phonème initial-final d'un mot

- substituer des phonèmes pour construire des mots

- soustraire des phonèmes pour construire des mots

- additionner des phonèmes pour construire des mots

- Quatrième axe : phonie-graphie

- Etablir des liens entre la phonie et la graphie

- Identifier les lettres muettes dans un mot.

\section{9}




\section{- La grille des compétences de la compréhension orale:}

\section{But :}

Cette grille vise à déterminer les compétences de la compréhension orale nécessaires aux élèves de la deuxième année préparatoire en français deuxième langue étrangère dans les écoles officielles des langues.

\section{$\checkmark$ Les sources de la grille:}

Pour préparer cette grille, le chercheur a eu recours à des sources multiples:

- L'étude théorique;

- Aussi quelques ouvrages pertinents :

- Carlette, E. (2001) : Mieux apprendre à comprendre l'oral en langue étrangère dans le français dans le monde. Recherches et applications. Janvier, CLE, Paris

- Cornaire, C. \& Germain, C. (1998): La compréhension orale. CLE International .ISBN 2-09-033327- 8, Ed. Marie-Christine, Paris.

- Gina, L. (2012) : L'enseignement, renforcement linguistique, la compréhension orale, IFADEM, ministère de l'éducation nationale et de la formation, Haïti.

\section{$\checkmark$ La description :}

La forme initiale de la grille des compétences de la compréhension orale comprend 17 compétences. Ce sont les compétences qui peuvent jouer un rôle crucial, du point de vue du chercheur, à développer la compréhension orale chez l'échantillon de la recherche.

Après avoir élaboré la grille des compétences de la compréhension orale, nous l'avons présenté, sous une forme initiale, aux membres du jury afin qu'ils :

- Vérifient la justesse de la formulation des compétences de la compréhension orale, et de les modifier si nécessaire.

- Suppriment les compétences non nécessaires.

- Rajoutent les compétences non inclus.

- Vérifient que les compétences sont du niveau des élèves de la deuxième année préparatoire qui étudient le français deuxième langue étrangère dans les écoles officielles des langues.

Les membres du jury ont apprécié la grille des compétences de la compréhension orale à l'exception de quelques remarques concernant la formulation. Ils ont supprimé les items numéro 5-8-9-12-13-16. Ces

\section{0}


items ont été supprimés parce que certains sont inclus implicitement dans les autres compétences et les autres sont répétés ou incompatible avec le niveau de l'échantillon. Les membres du jury ont aussi modifié les items numéro 3-4-6.

\section{$\checkmark$ Le calcul des pourcentages des compétences:}

Les compétences les plus importantes à développer chez les élèves d'après le jury sont onze compétences. Ce sont les compétences qui sont plus de $75 \%$.

Prenant en considération ces remarques, et faisant ces modifications, la grille a pris sa forme finale qui contient les compétences suivantes:

- Saisir le sens global du texte sonore.

- Saisir les sens des mots.

- Identifier des synonymes des mots du texte sonore.

- Déterminer des antonymes des mots du texte sonore.

- Repérer les idées essentielles du texte sonore.

- Repérer les idées secondaires du texte sonore.

- Identifier les personnages cités au texte sonore.

- Identifier le genre du texte (par. ex. lettre, article, publicité. etc).

- Proposer un titre adéquat du texte.

- Saisir l'intention de l'auteur.

- Identifier les évènements dans un texte sonore.

- Un pré/post test de la conscience phonologique.Pour élaborer ce test, nous avons suivi les étapes suivantes :

\section{$\checkmark$ But du test :}

Ce test vise à mesurer les compétences de la conscience phonologique chez les élèves de la deuxième année préparatoire qui étudient le français deuxième langue étrangère dans les écoles officielles des langues.

\section{$\checkmark$ Les sources du test :}

Pour élaborer ce test, le chercheur a consulté les ouvrages suivants :

- Desrochers, A., Kirby, J. R., Thompson, G. L., \& Fréchette, S. (2009). Le rôle de la conscience phonologique dans l'apprentissage de la lecture. Revue du Nouvel-Ontario, (34), 59-82.

- Guelenne Ch., (2013) : La Conscience Phonologique : Dossier d'Activités, les Planabums, Retz.

\section{1}


- Valette, R. (1975) Le test en langue étrangère, Trad. Par Francis Rey, Paris: Hachette,

- Milanovic, M. (2002). Évaluation de compétences en langues et conception de tests. Division des Politiques Linguistiques. Éditions du Conseil de l'Europe. Strasbourg.

\section{$\checkmark$ La description:}

Ce test se compose de dix-sept questions. Chaque question contient cinq items. La totalité des notes du test est 80 points et la durée est 90 minutes. Ces questions visent à mesurer les capacités des élèves à segmenter, compter, repérer, substituer, soustraire et additionner, un mot, une syllabe, un phonème et aussi sa capacité à relier la relation phonie-graphie.

\section{$\checkmark$ La transcription et le barème du test:}

Pour la transcription et le barème du test (Voir annexe 5).

\section{$\checkmark$ La fidélité du test:}

Pour calculer la fidélité du test, on a choisi un échantillon de 30 élèves de la deuxième année préparatoire. Un intervalle de deux semaines a séparé les deux applications du pré-post test. Pour calculer la fidélité, on a appliqué la formule de coefficient de corrélation de Pearson.

Le coefficient de la fidélité est 0.91. Ce qui éprouve la fidélité du test.

\section{$\checkmark$ La validité du test:}

Pour calculer la validité du test, on a appliqué la formule suivante: La Validité $=\sqrt{ }$ La fidélité

- Un pré/post test de la compréhension orale.

Pour élaborer ce test, nous avons suivi les étapes suivantes :

\section{$\checkmark$ But du test:}

Ce test vise à mesurer les compétences de la compréhension orale chez les élèves de la deuxième année préparatoire qui étudient le français deuxième langue étrangère dans les écoles officielles des langues.

\section{$\checkmark$ Les sources du test:}

Pour élaborer ce test, le chercheur a consulté les ouvrages suivants :

\section{2}


- Valette, R. (1975) Le test en langue étrangère, Trad. Par Francis Rey, Paris: Hachette,

- Bolton; S. (1987) Evaluation de la compétence communicative en langue étrangère, collection L.A.L., Paris: Hatier,

- Milanovic, M. (2002). Évaluation de compétences en langues et conception de tests. Division des Politiques Linguistiques. Éditions du Conseil de l'Europe. Strasbourg.

- Bachman, L. F., \& Palmer, A. (1996). Language testing in practice: Designing and developing useful language tests. New York: Oxford University Press.

\section{$\checkmark$ La description:}

Ce test se compose de cinq questions de types ; choix multiples, questions à trous, questions d'appariement et vrai/faux. La totalité des notes du test est 80 points et la durée est 60 minutes.

\section{$\checkmark$ La transcription et le barème du test:}

Pour la transcription et le barème du test (Voir annexe 4).

\section{$\checkmark$ La fidélité du test:}

Pour calculer la fidélité du test, on a choisi un échantillon de 30 élèves de la deuxième année préparatoire. Un intervalle de deux semaines a séparé les deux applications du pré-post test. Pour calculer la fidélité, on a appliqué la formule de coefficient de corrélation de Pearson.

Le coefficient de la fidélité est 0.91. Ce qui éprouve la fidélité du test.

\section{$\checkmark$ La validité du test:}

Pour calculer la validité du test, on a appliqué la formule suivante: La Validité $=\sqrt{ }$ La fidélité

$$
\mathrm{V}=\sqrt{\mathrm{F}} \quad \mathrm{F}=\text { Fidélité }
$$

La Validité= ,91

- Deuxièmement : 2. les outils du traitement expérimental :

Les outils du traitement se composent d'un programme élaboré à la lumière de stratégie de la classe inversée.

\section{3}


Pour élaborer les outils du traitement, nous avons passé par les étapes suivantes:

\section{- Le but du programme:}

Ce programme vise à développer la conscience phonologique chez les élèves de la deuxième année préparatoire dans les écoles officielles de la langue (école d'Ahmed zoweil) au gouvernorat de Kalyobyia et à vérifier son effet sur les compétences de la compréhension orale chez ces élèves.

\section{- Le choix du contenu et description:}

Le chercheur a élaboré un programme en fonction de stratégie de la classe inverse qui contient quatre unités.

Pour choisir le contenu adéquat de ce programme, nous avons pris en considération les principes suivants:

$\checkmark$ Que ce contenu traduise les besoins langagiers des élèves. Nous avons choisi ce contenu en fonction des objectifs déjà précisés.

$\checkmark$ Que la langue utilisée soit au niveau des élèves.

$\checkmark$ Que ce contenu soit présenté sous forme d'activités permettant aux apprenants d'apprendre selon leurs rythmes et leurs capacités et de se collaborer pour atteindre l'objectif visé qui est le développement des compétences de la conscience phonologique et celles de la compréhension orale.

Le programme choisi comprend quatre unités construites à la lumière de la classe inversée. Chaque unité comprend trois textes sonores suivis par plusieurs activités concernant la conscience phonologique.

\section{- La préparation des activités d'apprentissage.}

Les activités proposées ont mis l'accent sur le rôle positif et actif de l'élève. Elles sont concentrées sur les élèves et sur ce qu'ils doivent faire pour atteindre les objectifs de l'enseignement.

Ces activités sont complémentaires à la méthode Bienvenue (2) visée aux élèves de la deuxième année préparatoire aux écoles officielles des langues, dont «l'objectif est de faire acquérir à l'élève une compétence de communication, consolidée par des acquisitions linguistiques progressives, ainsi qu'une compétence pratique et culturelle ». (Bienvenue 2, 1993, p. 6)

\section{4}


Ces activités se varient selon l'étape de l'apprentissage comme suit:

$\checkmark$ Les activités de préparation :

Il existe plusieurs façons de préparer les apprenants, on peut:

- Leur annoncer quelle texte, ils vont écouter, (par exemple: chez le médecin, à la gare, entretien d'embauche..etc.)

- Leur communiquer les informations de référence nécessaires.

- Les prévenir qu'ils ne comprendront pas tout à la première écoute ; il s'agit d'abord de saisir l'idée générale du texte sonore, puis de comprendre les détails.

- Leur poser quelques questions pour motiver les élèves à collaborer.

- Engager une discussion sur le thème du texte, leur donnant ainsi l'occasion d'exprimer leurs opinions et leurs sentiments.

\section{$\checkmark$ Activités de progrès:}

Les apprenants pourraient:

- Exécuter un jeu de rôles avec ses pairs.

- Ecouter un enregistrement puis répondre aux questions propres à cet enregistrement.

\section{- Questions :}

- choix multiple

- vrai/faux

- phrases à trous

- questions d'appariement

$\checkmark$ La description et la répartition des activités:

Le programme contient quatre unités. Chaque unité comprend trois texte sonores suivis par des activités portées sur la conscience phonologique.

\section{- Chaque activité comprend:}

- L'objectif de l'activité.

- Le matériel nécessaire (s 'il existe).

- Le déroulement de l'activité.

- Le mode de travail: individuellement, en petit groupe ou en groupe classe.

- les supports: Les supports utilisés à l'enseignement des quatre unités sont:

\section{5}


$\checkmark$ Une disquette sur laquelle est enregistré le contenu de quatre unités qui forme le programme (texte sonore, conversations audio, vidéos, ...etc.).

$\checkmark$ Un ordinateur.

$\checkmark$ Un écran.

$\checkmark$ Un appareil de data show.

$\checkmark$ Un tableau blanc. Un CD (disquette) pour travailler à la maison,

$\checkmark$ Le public visé:

Ce programme s'adresse aux élèves de la deuxième année préparatoire aux écoles officielles des langues.

\section{$\checkmark$ La méthode d'enseignement:}

La méthode adoptée dans cette recherche dépend des stratégies d'apprentissage :

- Auto-apprentissage.

- auto- évaluation.

- jeu de rôles.

\section{$\checkmark$ L'évaluation:}

- Formative: pendant le cours suivi de feedback.

- Sommative: les post tests de la conscience phonologique et de la compréhension orale

\section{- Troisièmement : 3- l'expérimentation:}

\section{$\checkmark$ L'échantillon :}

Nous avons choisi notre échantillon parmi les élèves de la deuxième année préparatoire de l'école Ahmed Zoweil officielle des langues au gouvernorat de Qualyobyia.

Ces élèves étudient le français comme 2ème langue étrangère d'après la méthode «Bienvenue 2».

Les membres de l'échantillon est 60 élèves répartis en deux groupes : 30 comme groupe expérimental et 30 comme groupe témoin.

\section{$\checkmark$ Le plan temporel de l'expérimentation}

L'expérimentation a commencé du 2 octobre 2015 par les pré-tests et a terminé au 2 décembre 2015 par les post tests. L'application des activités du programme a duré presque deux mois, à raison de deux cours par semaine.

Le tableau suivant montre la distribution des séances de l'enseignement des unités.

\section{6}


Tableau no. (6)La distribution des heures de l'enseignement

\begin{tabular}{|c|c|c|}
\hline Types de séances & Nombre de séances & Nombre d'heures \\
\hline séance de préparation & 1 & 2 \\
\hline Premier unité & 4 & 6 \\
\hline Deuxième unité & 4 & 6 \\
\hline Troisième unité & 4 & 6 \\
\hline Quatrième unité & 4 & 6 \\
\hline total & 17 séances & 26 \\
\hline
\end{tabular}

\section{L'application des pré-tests.}

Nous avons appliqué les pré-tests sur l'échantillon de la recherche (les deux groupes) le 2 octobre 2015. L'application du test de la compréhension orale a duré 60 minutes mais, le test de la conscience phonologique a duré 80 minutes.

$\checkmark$ Les pré-tests (la compréhension orale et la conscience phonologique).

Après avoir appliqué les deux pré-tests, nous avons corrigé les réponses des élèves. Le résultat a indiqué que:

- Les élèves de la deuxième année préparatoire sont faibles dans la compréhension orale et la conscience phonologique.

- Il n'existe pas de différence significative entre les élèves des deux groupes (expérimental et témoin) avant l'application de l'outil du traitement (le programme).

Tableau no. (5)La différence entre le groupe expérimental et le groupe témoin aux pré-tests de la compréhension orale et de la conscience phonologique

\begin{tabular}{|c|c|c|c|c|c|c|}
\hline Groupe & test & $N$. & $M$. & E.T. & $T$. & $S$. \\
\hline expérimental & \multirow{2}{*}{$\begin{array}{c}\text { Conscience } \\
\text { phonologique }\end{array}$} & 30 & 36,93 & 6,27 & \multirow{2}{*}{0,277} & \multirow{2}{*}{ N.S. } \\
\hline Témoin & & 30 & 36,53 & 4,81 & & \\
\hline expérimental & \multirow{2}{*}{$\begin{array}{c}\text { Compréhension } \\
\text { orale }\end{array}$} & 30 & 36,96 & 4,05 & \multirow{2}{*}{1,32} & \multirow{2}{*}{ N.S. } \\
\hline Témoin & & 30 & 35,63 & 3,73 & & \\
\hline
\end{tabular}

N. Nombre des élèves

E. T. Ecart type

M. Moyenne des notes

S. Niveau de signification

Ce tableau montre qu'il n'existe pas de différences significatives entre les moyennes des notes des élèves du groupe expérimental et celles des élèves du groupe témoin aux pré-tests de la conscience phonologique et de la compréhension orale.

La valeur de $T$. pour le test de la conscience phonologique est $(0,277)$ et pour le test de la compréhension orale est $(1,32)$. Ces valeurs ne sont pas significatives. Ces résultats indiquent que les élèves de deux groupes sont homogènes avant l'application de l'outil du traitement (le

\section{7}


programme) dans les compétences de la conscience phonologique et celles de la compréhension orale.

\section{$\checkmark$ L'application des post-tests}

Après avoir terminé l'application du programme, nous avons appliqué les post-tests (de la conscience phonologique et de la compréhension orale) le 12 octobre 2015. Nous avons corrigé les réponses des élèves. Nous avons calculé les moyennes et les écarts types pour les utiliser à l'analyse statistique.

\section{- Quatrièmement : Analyse et discussion des résultats}

Pour vérifier l'effet du développement de la conscience phonologique sur l'amélioration des compétences de la compréhension orale, le chercheur va exposer les résultats et l'analyse statistique à travers la vérification des hypothèses de la recherche. L'analyse statistique se fait par le programme SPSS.

Les cinq hypothèses de cette recherche sont vérifiées par le T. Test. Ce test calcule la différence entre les moyens des notes entre deux groupes.

\section{- Première hypothèse}

Il existe une différence significative entre les moyennes des notes des élèves du groupe expérimental et celles du groupe témoin au post test de la conscience phonologique en faveur du groupe expérimental.

Pour vérifier cette hypothèse, le chercheur a calculé la valeur de T. pour examiner la signification statistique des différences entre les deux groupes (expérimental et témoin) au post test de la conscience phonologique.

Tableau no. (1)La différence entre les moyennes des notes des élèves du groupe expérimental et celles des élèves du groupe témoin au post test de la conscience phonologique.

\begin{tabular}{|c|c|c|c|c|c|}
\hline Axes du test & groupes & $M$ & $E . T$. & $T$. & $S$. \\
\hline \multirow{2}{*}{ Syllabes } & Groupe expérimental & 23,46 & 2,27 & \multirow{2}{*}{13,73} & \multirow{2}{*}{0,01} \\
\hline & Groupe témoin & 14,30 & 2,86 & & \\
\hline \multirow{2}{*}{ Phonèmes } & Groupe expérimental & 22,90 & 3,15 & \multirow{2}{*}{10,56} & \multirow{2}{*}{0,01} \\
\hline & Groupe témoin & 14,20 & 3,22 & & \\
\hline \multirow{2}{*}{ Rimes } & Groupe expérimental & 7,66 & 1,51 & \multirow{2}{*}{9,32} & \multirow{2}{*}{0,01} \\
\hline & Groupe témoin & 4,23 & 1,33 & & \\
\hline \multirow{2}{*}{$\begin{array}{l}\text { Phonie- } \\
\text { graphie }\end{array}$} & Groupe expérimental & 7,96 & 1,37 & \multirow{2}{*}{11,33} & \multirow{2}{*}{0,01} \\
\hline & Groupe témoin & 4,40 & 1,037 & & \\
\hline \multirow{2}{*}{ Total } & Groupe expérimental & 62 & 4,73 & \multirow{2}{*}{19,69} & \multirow{2}{*}{0,01} \\
\hline & Groupe témoin & 37,13 & 5,04 & & \\
\hline
\end{tabular}

$N=$ Nombre d'élèves

$M=$ Moyenne des notes

E.T = Ecart type

$S=$ Niveau de signification 
Ce tableau montre qu'il existe des différences significatives entre les moyennes des notes des élèves du groupe expérimental et celles des élèves du groupe témoin au post test de la conscience phonologique en faveur du groupe expérimental. Donc, ces différences se trouvent dans tous les niveaux de la conscience phonologique. La valeur de T. dans le test total est $(19,69)$. Cette valeur est significative au niveau 0.01.

Cela indique aussi que le niveau des élèves est plus élevé au post test qu'au pré test dans tous les niveaux de la conscience phonologique (syllabes- phonèmes- Rimes- phonie-graphie). Donc la première hypothèse est confirmée.

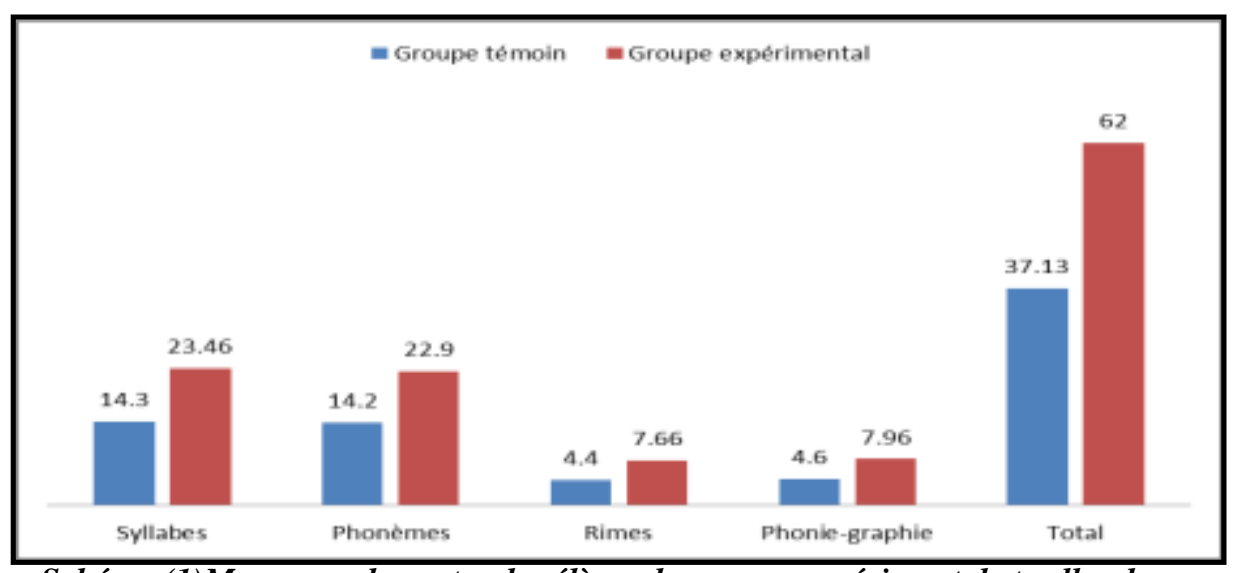

Schéma (1)Moyennes des notes des élèves du groupe expérimental et celles des élèves du groupe témoin au post test de la conscience phonologique

- Deuxième hypothèse

Il existe une différence significative entre les moyennes des notes des élèves du groupe expérimental au pré-post test de la conscience phonologique en faveur du post test.

Pour vérifier cette hypothèse, le chercheur a calculé la valeur de T.

Tableau no. (2)La différence entre les moyennes des notes des élèves du groupe expérimental au pré-post test de la conscience phonologique.

\begin{tabular}{|c|c|c|c|c|c|c|}
\hline Axes du test & groupes & $N$ & $M$ & E.T. & $T$. & $S$. \\
\hline \multirow{2}{*}{ Syllabes } & Post-test & 30 & 23,46 & 2,27 & \multirow{2}{*}{19,02} & \multirow[b]{2}{*}{0,01} \\
\hline & Pré-test & 30 & 14 & 3,26 & & \\
\hline \multirow{2}{*}{ Phonèmes } & Post-test & 30 & 22,90 & 3,15 & \multirow{2}{*}{21,10} & \multirow{2}{*}{0,01} \\
\hline & Pré-test & 30 & 14,23 & 3,30 & & \\
\hline \multirow{2}{*}{ Rimes } & Post-test & 30 & 7,66 & 1,51 & \multirow{2}{*}{20,88} & \multirow{2}{*}{0,01} \\
\hline & Pré-test & 30 & 4,10 & 1,51 & & \\
\hline \multirow{2}{*}{$\begin{array}{l}\text { Phonie- } \\
\text { graphie }\end{array}$} & Post-test & 30 & 7,96 & 1,37 & \multirow{2}{*}{27,57} & \multirow{2}{*}{0,01} \\
\hline & Pré-test & 30 & 4,60 & 1,30 & & \\
\hline \multirow{2}{*}{ Total } & Post-test & 30 & 62 & 4,73 & \multirow{2}{*}{34,18} & \multirow{2}{*}{0,01} \\
\hline & Pré-test & 30 & 36,93 & 6,27 & & \\
\hline
\end{tabular}



$N=$ Nombre d'élèves
$M=$ Moyenne de notes
$T=$ Valeur de $T$.
E. $T=$ Ecart type
$S=$ Niveau de signification

Ce tableau montre qu'il existe une différence significative entre les moyennes des notes des élèves du groupe expérimental au pré-post test de la conscience phonologique en faveur du post test. La valeur de T. est (34.18). Cette valeur est significative au niveau 0,01.

Cela indique que le niveau des élèves est plus élevé au post test qu'au pré test dans tous les niveaux de la conscience phonologique (syllabesphonèmes- Rimes- phonie-graphie). Donc la deuxième hypothèse est confirmée.

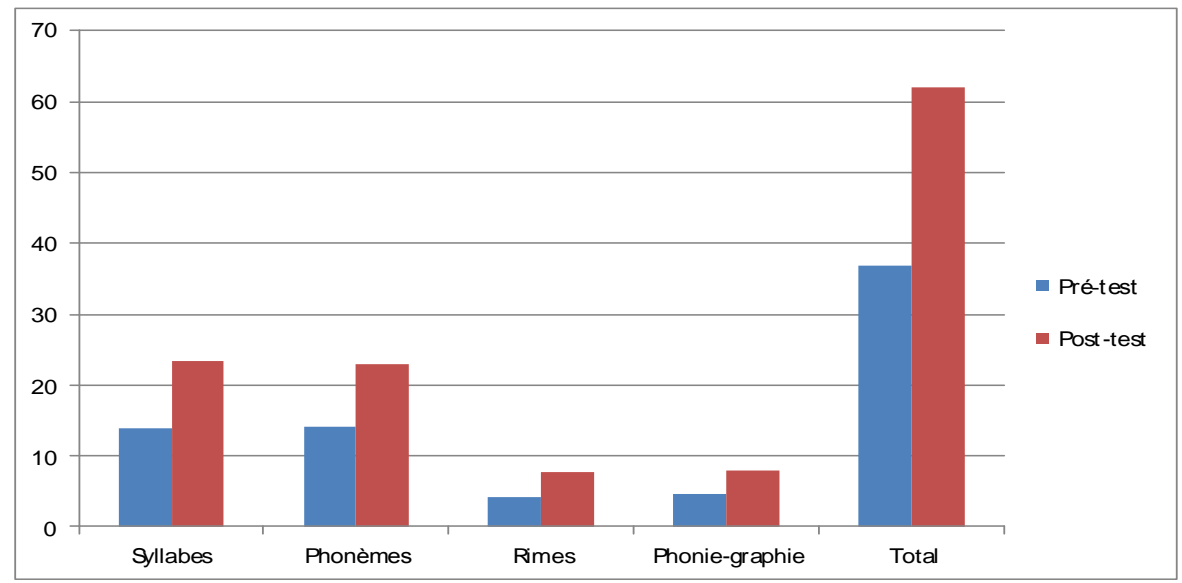

Schéma (2)Moyennes des notes des élèves du groupe expérimental au pré-post test de la conscience phonologique

\section{- Troisième hypothèse}

Il existe une différence significative entre les moyennes des notes des élèves du groupe expérimental et celles du groupe témoin au post test de la compréhension orale en faveur du groupe expérimental.

Pour vérifier cette hypothèse, le chercheur a calculé la valeur de T.

Tableau no. (3)La différence entre les moyennes des notes des élèves du groupe expérimental et celles des élèves du groupe témoin au post test de la compréhension orale.

\begin{tabular}{|c|c|c|c|c|c|}
\hline Groupe & $N$. & $M$. & E.T. & $T$. & $S$. \\
\hline expérimental & 30 & 66,70 & 5,47 & 22,054 & 0.01 \\
\hline témoin & 30 & 36,80 & 5,01 & & \\
\hline
\end{tabular}



$N=$ Nombre d'élèves
$M=$ Moyenne des notes
E.T $=$ Ecart type
$S=$ Niveau de signification
$T=$ Valeur de $T$.

Ce tableau montre qu'il existe des différences significatives entre les moyennes des notes des élèves du groupe expérimental et celles des élèves du groupe témoin au post test de la compréhension orale en faveur du groupe expérimental. La valeur de T. est (22,054). Cette valeur est significative au niveau 0.01. Donc la troisième hypothèse est confirmée.

\section{Test de la compréhension orale}

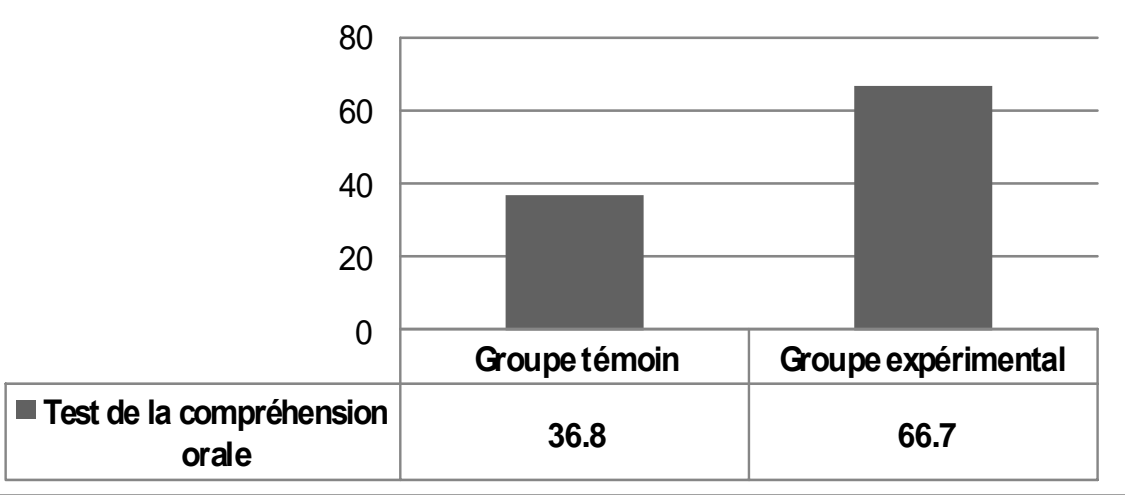

Schéma (3)Moyennes des notes des élèves du groupe expérimental et celles des élèves du groupe témoin au post test de la compréhension orale

\section{- Quatrième hypothèse}

Il existe une différence significative entre les moyennes des notes des élèves du groupe expérimental au pré-post test de la compréhension orale en faveur du post test.

Pour vérifier cette hypothèse, le chercheur a calculé la valeur de T.

Tableau no. (4)La différence entre les moyennes des notes des élèves du groupe expérimental au pré-post test de la compréhension orale.

\begin{tabular}{|c|c|c|c|c|c|}
\hline Groupe Expérimental & $N$. & M. & E.T. & $T$. & $S$. \\
\hline Pré test & 30 & 66,70 & 5,47 & \multirow{2}{*}{28,074} & \multirow{2}{*}{0.01} \\
\hline Post test & 30 & 36,96 & 4,05 & & \\
\hline
\end{tabular}

\section{1}


Ce tableau montre qu'il existe une différence significative entre les moyennes des notes des élèves du groupe expérimental au pré-post test de la compréhension orale en faveur du post test. La valeur de T. est (28,074). Cette valeur est significative au niveau 0,01.

Cela indique que le niveau des élèves est plus élevé au post test qu'au pré test dans toutes les compétences de la compréhension orale Donc la quatrième hypothèse est confirmée.

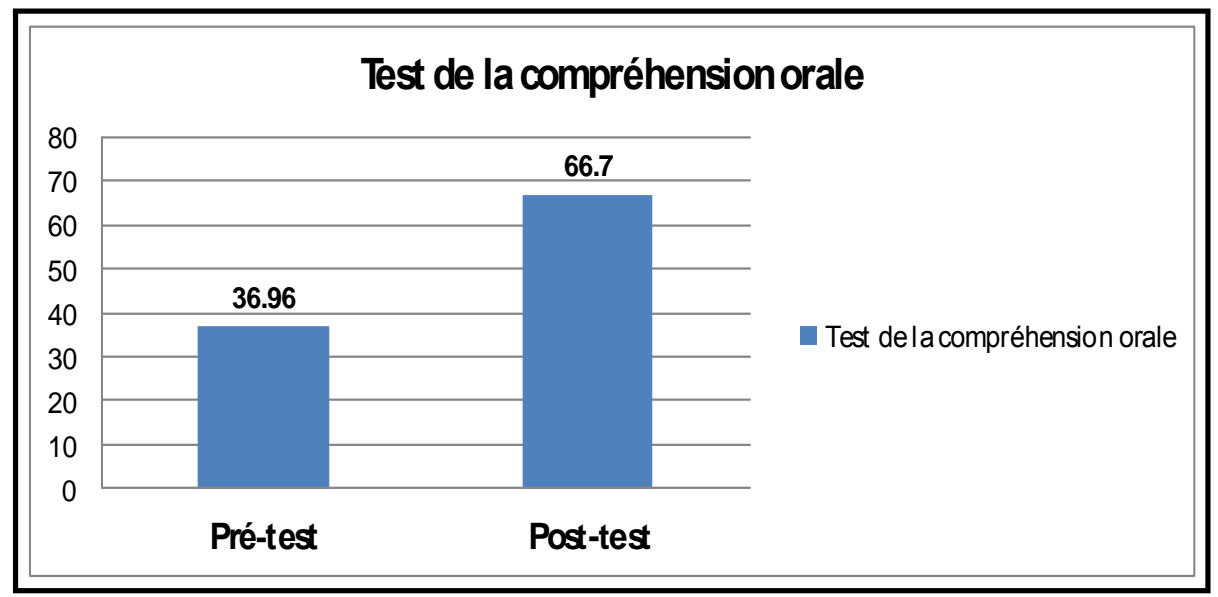

Schéma (4) Moyennes des notes des élèves du groupe expérimental au pré-post test de la compréhension orale

\section{- Cinquième hypothèse}

Il existe une relation entre le développement des compétences de la conscience phonologique et celles de la compréhension orale chez les élèves de la deuxième année préparatoire

Pour vérifier cette hypothèse, le chercheur a calculé le coefficient de corrélation aux post-tests pour vérifier la relation entre le développement des compétences de la conscience phonologique et celles de la compréhension orale.

Tableau no. (5) Relation entre le développement des compétences de la conscience phonologique et celles de la compréhension orale.

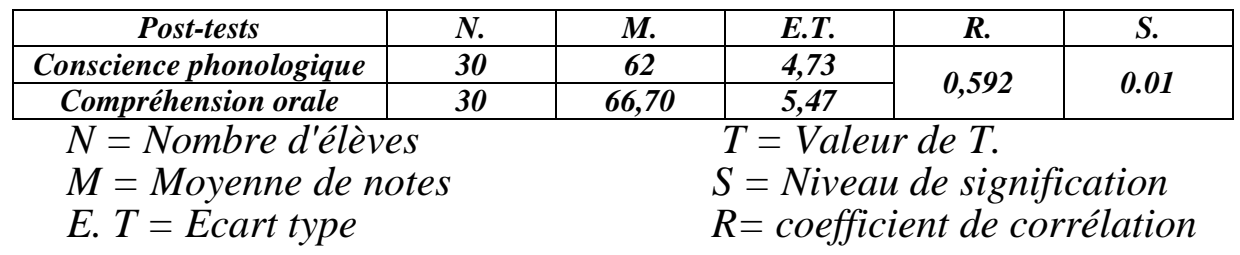

\section{2}


Ce tableau montre que la valeur du coefficient de corrélation entre les notes des élèves dans les post-tests de la conscience phonologique et de la compréhension orale est significative.

La valeur du coefficient de corrélation est (0,592). Cette valeur est significative au niveau 0,01.

Cela indique qu'il y a une relation entre le développement des compétences de la conscience phonologique et celles de la compréhension orale chez les élèves de la deuxième année préparatoire. Cette relation signifie que le développement de la conscience phonologique a amené à développer la compréhension orale. Donc la cinquième hypothèse est confirmée.

\section{- Discussion des résultats:}

D’après les résultats déjà présentés, nous pouvons conclure que la classe inversée est efficace à développer les compétences de la conscience phonologique chez l'échantillon de la recherche. Il est à noter que le développement de la conscience phonologique a amené à développer la compréhension orale.

- La classe inversée a contribué à créer une atmosphère interactive qui favorise l'acquisition des compétences de la conscience phonologique dans un contexte plus naturel et plus interactif. Les élèves étaient donc plus enclins à apprendre et à fournir l'effort nécessaire.

- La classe inversée a contribué à favoriser l'auto-apprentissage l'apprentissage autonome chez les élèves.

- La classe inversée a aidé à développer des attitudes positives envers la matière enseignée ce qui a rendu les élèves plus motivés et a maximisé leur acquisition des compétences de la conscience phonologique.

- le feedback automatisé, systématisé et direct que les élèves ont reçu, les a aidés à se corriger pendant le travail et à se communiquer efficacement.

- La variété des exercices présentés aux élèves, leur a permis de pratiquer la langue dans ses différents aspects.

- Recommandations de la recherche :

D’après les résultats de la présente étude, nous pouvons présenter les recommandations suivantes:

- Le développement de la conscience phonologique a besoin d'une pratique. Les élèves doivent participer à des situations réelles, comme dans la vie quotidienne.

\section{3}


- La nécessité d'insérer la conscience phonologique dans les programmes du français 2ème langue étrangère comme pré-requis des compétences orales.

- L’élève doit être un acteur dans ses apprentissages et non seulement un spectateur pour être capable d'apprendre de façon autonome.

- La nécessité d'utiliser les méthodes modernes appliquées dans le domaine de l'apprentissage des langues étrangères qui reposent sur l'emploi du support audio-visuel (cassette audio - dessin - image carte, etc...). La variété des techniques aide les élèves à bien apprendre.

- La nécessité de donner la priorité à l'oral et particulièrement à la compréhension et l'expression orales.

- La nécessité d'accorder un souci particulier à la compréhension orale en classe FLE en entrainant les enseignants et les futurs enseignants de FLE aux étapes d'enseignement de la compréhension orale et en faisant de la compréhension orale une partie des tests du français dans nos écoles.

- La nécessité d'élaborer des programmes basés sur la classe inversée visant à développer les compétences orales et écrites chez les élèves dans tous les cycles d'apprentissage.

\section{- Références}

- Aouina, M. (2008). L'enseignement / apprentissage de la compréhension orale par le biais d'un document sonore, (Mémoire de maitrise). Faculté des lettres et des sciences humaines, Université El-hadj Lakhdar de BATNA, Algérie.

- Becchetti-Bizot, C., Houzel, G., \& Taddei, F. (2017). Vers une société apprenante. Rapport sur la recherche et développement de l'éducation tout au long de la vie. Repéré à http://cache. media. education. gouv . fr/ file/ 2017/ 40/ 3/ Rapport_recherche et developpement_education_V2_756403.pdf.

- Berberian, N. (2016). La classe inversée en FLE : Création d'une formation. Mémoire Master 2 LFA., inédit, Madrid Complutense Paris IV Sorbonne.

- Bissonnette, S. \& Gauthier, C. (2013). Faire la classe à l'endroit ou à l'envers? Formation et profession - Revue scientifique internationale en éducation, Québec

\section{4}


- Bouchlion, D. (2014). Mise en cuvre de la classe inversée et de l'évaluation choisie Collège Aliénor d'Aquitaine 22 ROUTE DU MARTINET , 33770 SALLES

- Bricard, E. (1996). La passion d'une langue vivante, Paris: L'Harmattan

- Briquet-Duhazé, S., \& Rezrazi, A. (2014). Résultat d'un entraînement en conscience phonologique chez des élèves en difficultés de lecture au cycle 3. Enfance, (2), 119-134.

- Caroline Di Miceli \& Jean-Pierre. (2007). De la reconstruction du sens en compréhension orale : difficultés et stratégies. Académie de Montpellier, thèse de magistère, Université de Montpellier.

- CECR (2001). Cadre européen commun de référence pour les langues. Conseil de l'Europe /Les Editions Didier, Paris.

- Cornaire, C. \& Germain, C. (1998). La compréhension orale. CLE International, Paris.

- Cuq, J.P. \& Gruca, I. (2005). Cours de didactique du français langue étrangère et seconde. Grenoble: Presses universitaires de Grenoble.

- Dargnat, M. (2006). L'oral comme fiction, thèse de doctorat, Université de Provence - Aix-Marseille I; Université de Montréal.

- Demont, E. \& Gombert, JE. (1996). Phonological awareness as a predictor of recoding skills and syntactic awareness as a predictor of comprehension skills, British journal of educational psychology, V.66 (3), 315-332

- Descazaux, S. (2009). Conscience phonologique, 14 /1 /2009 http://www.acbordeaux.fr/ia40/fileadmin/pedagogie/circonscriptions /T/Animations_pedagogiques/Documents/compte_rendu_animation_ du_14-01-09.pdf (16/10/2014)

- Desrochers, A., Kirby, J. R., Thompson, G. L., \& Fréchette, S. (2009). Le rôle de la conscience phonologique dans l'apprentissage de la lecture. Revue du Nouvel-Ontario, (34), 59-82.

- Disponible sur http://www.inrp.fr/vst.

- Dufour, H. (2014). La classe inversée. Revue technologie 193, 44-47

- Dumont, A., \& Berthiaume, D. (2016). La pédagogie inversée : Enseigner autrement dans le supérieur par la classe inversée. De Boeck Supérieur.

- Feyfant, A., \& Gaussel, M. (2007). Méthodes de lecture et difficultés d'apprentissage. Dossier d'actualité Veille et Analyse, No. 31, Novembre.

\section{5}


- Galisson, R. \& Coste, D. (1976). Dictionnaire de Didactique des Langues, Hachette, Paris.

- Gaonac'h, D., \& Passerault, J.-M. (2006). Le langage. In J.-L. Roulin (Ed.), Psychologie cognitive (pp. 329-389). Rosny: Bréal.

- Gombert J.E (1990). Le développement métalinguistique, Paris PUF.

- González Ramos, S. (2016). La pédagogie inversée en cours de FLE. Université du Pays Basque

- Gremmo, M. J. et Holec, H. (1990). La compréhension orale : un processus et un comportement. Le Français dans le Monde, Recherches et Applications. $N^{\circ}$ spécial, Fév. /Mars, p.31-40.

- Guelenne Ch., (2013). La Conscience Phonologique : Dossier d'Activités, Les Planabums, Retz.

- Guichon, N. \& Nicolaev, V. (2011). Influence de certaines caractéristiques des tâches d'apprentissage sur la production orale en L2.Dans E, Nissen., F, Poyet. \&T, Soubrié, (coord.). Interagir et apprendre en ligne. Grenoble : Ellug. p. 61-76.

- Jean Jaurès. (2011), De l'écoute des sons à la conscience phonologique. Animation pédagogique, Hatier.

- Karima, F. (2009). La compréhension orale et les stratégies des élèves apprenant le français en 2eme année moyenne en Algérie. Université de Blida Algérie.

- Lebrun, M., \& Lecoq, J. (2015). Classes inversées. Enseigner et apprendre à l'endroit!

- Lecoq, J., Lebrun, M., \& Kerpelt, B. (2017). La classe à l'envers pour apprendre à l'endroit : Guide pratique pour débuter en classe inversée. les cahiers du lll $N 1$.

- Leduc, D., \& Mariez, J. M. (1990). Guide d'enseignement du français au secondaire. Volet II : Communication orale. Immersion

- Letarte, A \& Lapond, F. (2001). L'écoute en classe et la prise de notes. Université Laval. Centre d'organisation et de consultation psychologique, 4 e édition-Aout.

- Louise, M. (2005). Les domaines d'enseignement pédagogiques. Inspection Académique de l'Essonne. No.37, Octobre. Disponible sur l'internet à : http://www.bienlire education.fr

- Magnat, E. (2013). Le TBI comme instrument du développement de la conscience phonémique à l'école : une approche ergonomique. Linguistique. Thèse de doctorat, Université Stendhal - Grenoble.

\section{6}


- Marchal, B. (2015). De la classe enrichie en passant par la classe virtuelle, vers une classe inversée. Synergies Pays Riverains du Mékong, (7), 23-34.

- Mohammad, A.Ch. (2015). Emploi de La Conscience Phonographique Pour le Développement de la Compréhension Orale\& Pour le Renforcement des Rapports Phonèmes / Graphèmes en FLE Chez Les Maxi-Débutants, Revue de la lecture et de la connaissance, V.1, No.169.

- Morel, M. A., \& Danon-Boileau, L. (1998). Grammaire de l'intonation l'exemple du français. Editions Ophrys.

- Murieux, Ph. (1993). Pédagogies ESF. Editeur-Paris- 3eme édition ISBN: 27101-1036- 9, ISSN: 1158-4581.

- Popot, A. (2011). Le développement de la conscience phonologique et ses liens avec le langage écrit en classe de CP. Magistère, université D'Orléans.

- Rost, M. (2013). Teaching and researching: Listening. Routledge. London: Longman.

- Roy, N. (2014). La classe inversée : une pédagogie renversante ? Le tableau, 3(1), 1-2.

- Salem, A. (1992). Effet d'utilisation des méthodes communicatives sur les niveaux oral et écrit du français comme, deuxième langue étrangère, chez les élèves de la première secondaire, thèse de magistère, non publiée, faculté de pédagogie, université de Zagazig.

- Stanké, B. (2001). L’apprenti lecteur - Activités de conscience phonoloqiue. Montréal: Les Éditions de la Chenelière.

- Stéphanie Paquin, M.O.A. (2006) : la conscience phonologique

- Treiman, R., Clifton Jr, C., Meyer, A. S., \& Wurm, L. H. (2003). Language comprehension and production. Handbook of psychology, 525-547.

- Yagang, F. (1993) Listening problems and solutions. English Teaching Forum. Vol.31, N.2, January-march. 